\title{
Mean first passage times reconstruct the slowest relaxations in potential energy landscapes of nanoclusters
}

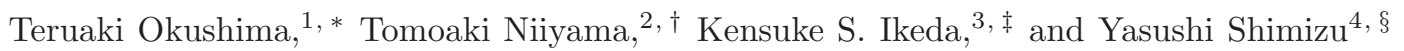 \\ ${ }^{1}$ College of Engineering, Chubu University, Matsumoto-cho, Kasugai, Aichi 487-8501, Japan \\ ${ }^{2}$ Graduate School of Natural Science and Technology, \\ Kanazawa University, Kakuma-cho, Kanazawa, Ishikawa 920-1192, Japan \\ ${ }^{3}$ College of Science and Engineering, Ritsumeikan University, \\ Noji-higashi 1-1-1, Kusatsu, shiga 525-8577, Japan \\ ${ }^{4}$ Department of Physics, Ritsumeikan University, \\ Noji-higashi 1-1-1, Kusatsu, shiga 525-8577, Japan
}

(Dated: September 25, 2019)

\begin{abstract}
Relaxation modes are the collective modes in which all probability deviations from equilibrium states decay with the same relaxation rates. In contrast, a first passage time is the required time for arriving for the first time from one state to another. In this paper, we discuss how and why the slowest relaxation rates of relaxation modes are reconstructed from the first passage times. As an illustrative model, we use a continuous-time Markov state model of vacancy diffusion in $\mathrm{KCl}$ nanoclusters. Using this model, we reveal that all characteristics of the relaxations in $\mathrm{KCl}$ nanoclusters come from the fact that they are hybrids of two kinetically different regions of the fast surface and slow bulk diffusions. The origin of the different diffusivities turns out to come from the heterogeneity of the activation energies on the potential energy landscapes. We also develop a stationary population method to compute the mean first passage times as mean times required for pair annihilations of particle-hole pairs, which enables us to obtain the symmetric results of relaxation rates under the exchange of the sinks and the sources. With this symmetric method, we finally show why the slowest relaxation times can be reconstructed from the mean first passage times.
\end{abstract}

\section{INTRODUCTION}

Recently, the dynamics of complex systems, such as the relaxation of glass-forming materials [1 12], the kinetics of biomolecules [13 23], and diffusion in nanoclusters 2430], were studied in a unified way for Markov state models [31 35]. The slowest relaxation modes of these systems describe the bottleneck processes, and hence they are the most crucial, e.g., for understanding glass transitions and rapid formations of mixed crystals [36 -43].

The relaxation rates and modes are the eigenvalues and eigenvectors, respectively, of the transition rate matrix of a Markov state model. In general, physical quantities are expressed in terms of the eigenvalues and eigenvectors. The resulting expressions, called spectral representations, give useful formulas that enable us to evaluate the physical quantities with use of the eigenvalues and eigenvectors [44 52]. We can compute the relaxation rates and modes of realistic, complicated Markov state models using numerical diagonalization algorithms [53]. However, it is hard to understand why the eigenvectors are formed in the shapes of the numerical diagonalization results because the eigenvectors are quite highdimensional and complicated. To extract the essence of the relaxation properties of Markov state models, there

\footnotetext{
* okushima@isc.chubu.ac.jp

$\dagger$ niyama@se.kanazawa-u.ac.jp

‡ ahoo@ike-dyn.ritsumei.ac.jp

$\S$ shimizu@se.ritsumei.ac.jp
}

have been many studies, such as lumping or renormalizing Markov state models [35 39, 42, 43], and applications of network algorithms, such as Dijkstra's shortest path algorithm 40]. Although there are many pioneering works concerning this problem [44-52], to the best of our knowledge, this problem has not yet been completely clarified.

As a more specific indicator of diffusive transport than the slowest relaxation rates, the first passage time is widely studied mainly for analyzing the kinetics in complex networks [15, 16, 54 58]. The first passage times from one state to another target state in a kinetic network are the required times of stochastic realizations for traveling from the former to the latter state for the first time. The corresponding mean first passage time is given by the mean value of the first passage times of the stochastic realizations.

Intuitively, we may interpret the slowest relaxation of a system as the process that transports the excess probability to the maximum probability states of the equilibrium distribution along the unavoidable and slowest transport routes in order to achieve the equilibrium distribution. Therefore, it may be possible to understand the formation of the slowest relaxation mode by searching for the states, where the first passage times to the maximum probability states are the largest, and then by finding out the main routes connecting the former to the latter states. To the best of our knowledge, however, there have been no such studies that search for the slowest relaxations with this idea. Instead, all pioneering works, e.g., Refs. [44 51], concern mainly how the mean first 
passage times are expressed with the relaxation modes via renewal theorems. It should be noted that in this paper we study the inverse problem, i.e., how and why the slowest relaxation modes are reconstructed by the mean first passage times.

As a realistic problem, we analyze a $\mathrm{KCl}$ nanocluster model having one vacancy [29, 30, 59]. The vacancy diffuses in the cluster and introduces the mixing of atoms in the cluster. As for the pioneering works on vacancy diffusion, the equilibrium vacancy concentration [60] and the relaxation process using a stochastic process simplified by a uniform diffusion equation [61] have been studied. Nevertheless, there are no studies in which the surface effects of nanoclusters on the relaxations of the vacancy diffusion are taken into consideration. The most substantial reason that makes such approaches difficult is that it is hard to estimate the transition rates between all adjacent states on the high-dimensional potential energy surface from interatomic interactions. Fortunately, in Ref. [30] we have successfully enumerated all states and all transition rates between adjacent states in nanoclusters of various sizes, and we elucidated the specific properties, such as migration energies of vacancies, arising from the surfaces of nanoclusters. In this paper, we use these transition data to construct the Markov state model of $\mathrm{KCl}$ nanoclusters and investigate the relationship between the slowest relaxation modes and the first passage times in the Markov state model equipped with the cluster surfaces of characteristic transition regions.

The purpose of this paper is twofold. One is to understand the formations of the slowest relaxation modes in terms of the first passage times of the vacancy diffusion in $\mathrm{KCl}$ nanoclusters. The other is to elucidate the theoretical basis for why such a mean first passage time analysis applies to the slowest relaxations.

In Sec. II, we introduce a Markov state model, its relaxation modes, and its mean first passage times in a general setting. For the mean first passage times, we develop a stationary population method that enables us to compute the first passage times from the stationary populations of the Markov state models that connect sinks with sources. In Sec. III we introduce the interatomic interaction of the $\mathrm{KCl}$ nanoclusters and then construct the Markov state models of the vacancy diffusion model. In Sec. IV] we compute the slowest relaxation mode and the mean first passage times of a $\mathrm{KCl}$ nanocluster. We find there that the shape of the energy landscape [32, 33] tells us why its relaxation makes effective use of the shortest routes of the vacancy from the center to the surface. In Sec. V] we study the second slowest relaxation mode and the corresponding mean first passage times of the $\mathrm{KCl}$ nanocluster. In Sec. VI, we first confirm that, under exchanging sinks and sources, the mean first passage time approximation for the relaxation times in Sec. IV is asymmetric, while that in Sec. $\mathrm{V}$ is suitably symmetric. We then develop a symmetric stationary population method for the mean first passage times, where they are interpreted as the mean first encounter times of particle- hole pairs. The iterative use of the symmetric method turns out to be equivalent to an inverse power method for diagonalization of matrices. We show that the mean first passage time approximations of the relaxation times are good approximations that converge to the exact relaxation times with the iterative use of the symmetric method.

\section{MARKOV STATE MODEL, RELAXATION RATES, AND MEAN FIRST PASSAGE TIMES}

In this section, we introduce a Markov state model, and we describe a popular method of calculating first passage times for this model according to Refs. 50, 52. We also show that the mean first passage times obey stationary population equations, which will be used to develop a symmetrized version of the population method later in Sec. VI]

\section{A. Continuous-time Markov state model}

We start with a continuous-time Markov state model described by a transition rate matrix $K$ with finite dimension, $n$, of the state space. The kinetic equation is given by

$$
\frac{d \boldsymbol{P}}{d t}=K \boldsymbol{P},
$$

where $\boldsymbol{P}$ is the probability distribution $\boldsymbol{P}=$ $\left(p_{1}, \ldots, p_{n}\right)^{T}$, with $p_{i}$ denoting the probability of the $i$ th state and the superscript $T$ denoting the transpose. We assume that $K$ is time-independent and satisfies $K_{i j} \geqslant 0$ $(i \neq j)$ and the probability conservation condition of $\sum_{i=1}^{n}(K)_{i j}=0(j=1,2, \ldots, n)$. Further, we assume that the equilibrium, $\lim _{t \rightarrow \infty} \boldsymbol{P}(t)$, is a unique vector $\boldsymbol{P}_{\text {eq }}$ satisfying the detailed balance conditions $(K)_{i, j}\left(\boldsymbol{P}_{\mathrm{eq}}\right)_{j}=$ $(K)_{j, i}\left(\boldsymbol{P}_{\text {eq }}\right)_{i}$ [50, 62]. Then, the eigenvalues of $K$ satisfy

$$
0=\lambda_{0}>\lambda_{1} \geqslant \cdots \geqslant \lambda_{n-1} .
$$

The equilibrium $\boldsymbol{P}_{\text {eq }}$ coincides with the zeroth eigenvector of $K$, and the first, second, $\ldots$ eigenvectors $\boldsymbol{P}_{i}$ of $K$ represent the slowest relaxation modes with the relaxation times of $\left|\lambda_{1}\right|^{-1} \geqslant\left|\lambda_{2}\right|^{-1} \geqslant \ldots$, respectively.

\section{B. Mean first passage times}

The mean first passage times, $t_{i, j}$, from a state $j$ to $i$ are evaluated by connecting perfect absorbers to all the final destinations of $i$. The resulting equation is given by

$$
\frac{d \boldsymbol{P}}{d t}=K \boldsymbol{P}-\boldsymbol{S}_{-},
$$

where $\boldsymbol{S}_{-}$represents the perfect absorbers that always keep $(\boldsymbol{P})_{i}=0$ for the sink states of $i$. Without the loss 
of generality, the sink states are assumed to be the states of $i=1, \ldots, m$, and the other states, which are free from the absorbers, are the remainders of $i=m+1, \ldots, n$. The perfect absorber conditions are represented as follows:

$$
\begin{gathered}
\boldsymbol{S}_{-}=\left(s_{1}, \ldots, s_{m}, 0, \ldots, 0\right)^{T} \equiv\left(\begin{array}{c}
\boldsymbol{s}_{-} \\
\mathbf{0}
\end{array}\right), \\
\boldsymbol{P}=\left(0, \ldots, 0, p_{m+1}, \ldots, p_{n}\right)^{T} \equiv\left(\begin{array}{c}
\mathbf{0} \\
\boldsymbol{p}
\end{array}\right) .
\end{gathered}
$$

By substituting Eqs. (4) and (5) for Eq. (3), we have the following solution with the initial condition of $\boldsymbol{P}_{0}=$ $\left(\begin{array}{c}0 \\ p_{0}\end{array}\right)$ :

$$
\begin{aligned}
& \boldsymbol{p}(t)=e^{t K_{F F}} \boldsymbol{p}_{0}, \\
& \boldsymbol{s}_{-}(t)=K_{S F} e^{t K_{F F}} \boldsymbol{p}_{0},
\end{aligned}
$$

where $\boldsymbol{p}_{0}$ satisfies

$$
\left\|\boldsymbol{p}_{0}\right\| \equiv \sum_{i=1}^{n-m}\left|\left(\boldsymbol{p}_{0}\right)_{i}\right|=\sum_{i=1}^{n-m}\left(\boldsymbol{p}_{0}\right)_{i}=1
$$

$K_{F F}$ is the submatrix with dimension $(n-m) \times(n-m)$ formed by selecting the rows of $K$ from $m+1$ to $n$ and the columns from $m+1$ to $n$, and $K_{S F}$ is the submatrix with dimension $m \times(n-m)$ formed by selecting the rows from 1 to $m$ and the columns from $m+1$ to $n$. The probability conservation property of the rate matrix of $K$ can be represented by

$$
\sum_{i=1}^{m}\left(K_{S F}\right)_{i j}+\sum_{i=1}^{n-m}\left(K_{F F}\right)_{i j}=0
$$

for $j=1,2, \ldots, n-m$. Multiplying both sides of Eq. (9) by $\left(K_{F F}^{-1}\right)_{j k}$ and adding the resultant equations from $j=$ 1 to $n-m$, we have the following equations

$$
\sum_{i=1}^{m}\left(-K_{S F} K_{F F}^{-1}\right)_{i k}=1 \quad(k=1,2, \ldots, n-m) .
$$

The $i$ th element of $s_{-}(t)$ describes the first passage time distribution of being absorbed in the $i$ th sink at time $t$. Therefore, by integrating Eq. (7) from $t=0$ to $\infty$, the probability of being absorbed in the $i$ th sink for $0 \leqslant t<\infty$ is given by the $i$ th element of

$$
\overline{\boldsymbol{s}}_{-}=\int_{0}^{\infty} \boldsymbol{s}_{-}(t) d t=-K_{S F} K_{F F}^{-1} \boldsymbol{p}_{0}
$$

where we use Eq. (7) and $\lim _{t \rightarrow \infty} e^{t K_{F F}}=0$, which holds because all eigenvalues of $K_{F F}$ are negative values. From Eq. (11) and $\left(\boldsymbol{s}_{-}(t)\right)_{i} \geqslant 0$, we see that $\left(\overline{\boldsymbol{s}}_{-}\right)_{i} \geqslant 0$. More- over, with the use of Eqs. (8) and (10), we have

$$
\begin{aligned}
\left\|\overline{\boldsymbol{s}}_{-}\right\| & \equiv \sum_{i=1}^{m}\left|\left(\overline{\boldsymbol{s}}_{-}\right)_{i}\right|=\sum_{i}\left(\overline{\boldsymbol{s}}_{-}\right)_{i} \\
& =\sum_{i}\left(-K_{S F} K_{F F}^{-1} \boldsymbol{p}_{0}\right)_{i} \\
& =\sum_{i, k}\left(-K_{S F} K_{F F}^{-1}\right)_{i k}\left(\boldsymbol{p}_{0}\right)_{k} \\
& =\sum_{k}\left(\boldsymbol{p}_{0}\right)_{k}=\left\|\boldsymbol{p}_{0}\right\|=1,
\end{aligned}
$$

whence

$$
\left\|\overline{\boldsymbol{s}}_{-}\right\|=\left\|\boldsymbol{p}_{0}\right\|=1 .
$$

The conditional probability distribution $\rho_{i}(t)$ of being absorbed at time $t$ when the system is known to be absorbed in the state of $i$ is given by

$$
\rho_{i}(t)=\frac{\left[\boldsymbol{s}_{-}(t)\right]_{i}}{\left(\overline{\boldsymbol{s}}_{-}\right)_{i}}=\frac{\left(K_{S F} e^{t K_{F F}} \boldsymbol{p}_{0}\right)_{i}}{\left(-K_{S F} K_{F F}^{-1} \boldsymbol{p}_{0}\right)_{i}} .
$$

Therefore, the mean first passage time, $t_{i, j}$, from the state $j$ to the sink state $i$ is given by

$$
\begin{aligned}
t_{i, j} & =\int_{0}^{\infty} t \rho_{i}(t) d t \\
& =\frac{\left(K_{S F} K_{F F}^{-2} \boldsymbol{p}_{0}\right)_{i}}{\left(-K_{S F} K_{F F}^{-1} \boldsymbol{p}_{0}\right)_{i}}
\end{aligned}
$$

with $\left(\boldsymbol{p}_{0}\right)_{k}=\delta_{k, j-m}(k=1,2, \ldots, n-m)$. Moreover, the mean first passage time, $t_{j}$, from the state $j$ to any absorbing states is given by

$$
\begin{aligned}
t_{j} & =\sum_{i}\left(\overline{\boldsymbol{s}}_{-}\right)_{i} t_{i, j}=\sum_{i}\left(K_{S F} K_{F F}^{-2} \boldsymbol{p}_{0}\right)_{i} \\
& =\sum_{i, k}\left(-K_{S F} K_{F F}^{-1}\right)_{i k}\left(-K_{F F}^{-1} \boldsymbol{p}_{0}\right)_{k} \\
& =\sum_{k}\left(-K_{F F}^{-1} \boldsymbol{p}_{0}\right)_{k},
\end{aligned}
$$

that is

$$
t_{j}=\left\|-K_{F F}^{-1} \boldsymbol{p}_{0}\right\|,
$$

where Eqs. (10), (11), and (14) are used.

Equations (14) and (15) are the basic formulas for calculating the mean first passage times.

Next, we show that the mean first passage times can be evaluated from a stationary population distribution. Let us consider the following mean residence time distribution,

$$
\overline{\boldsymbol{p}}=\int_{0}^{\infty} \boldsymbol{p}(t) d t=-K_{F F}^{-1} \boldsymbol{p}_{0},
$$

where $(\overline{\boldsymbol{p}})_{i}$ is the mean residence time in the $(i+m)$ th state for $i=1, \ldots, n-m$. Hence, the mean residence 
time in the whole system is given by the sum, $\|\overline{\boldsymbol{p}}\|$, of $(\overline{\boldsymbol{p}})_{i}$ from $i=1$ to $n-m$, which is, of course, equivalent to the mean first passage time $t_{j}$ of Eq. (15).

Equation (16) enables us to confirm that $\overline{\boldsymbol{p}}$ satisfies the following non-equilibrium stationary state equation:

$$
\frac{d \overline{\boldsymbol{p}}}{d t}=K_{F F} \overline{\boldsymbol{p}}+\overline{\boldsymbol{s}}_{+}=\mathbf{0}
$$

with $\overline{\boldsymbol{s}}_{+}=\boldsymbol{p}_{0}$. Hence, we can interpret $\overline{\boldsymbol{s}}_{+}$as the source term that adds one particle with distribution $\boldsymbol{p}_{0}$ per unit time, $\overline{\boldsymbol{p}}$ as the stationary population of Eq. (17), $\|\overline{\boldsymbol{p}}\|$ as the total population contained in $\overline{\boldsymbol{p}}$, and $\|\overline{\boldsymbol{p}}\|^{-1}$ as the probabilistic flow carried by one particle. Namely, we can also compute the mean first passage times as the total numbers, $\|\overline{\boldsymbol{P}}\|$, of particles in the stationary population $\overline{\boldsymbol{P}}$ obeying the following stationary equation:

$$
\frac{d}{d t} \overline{\boldsymbol{P}}=K \overline{\boldsymbol{P}}+\boldsymbol{S}_{+}-\boldsymbol{S}_{-}=\mathbf{0},
$$

where

$$
\overline{\boldsymbol{P}}=\left(\begin{array}{c}
\mathbf{0} \\
\overline{\boldsymbol{p}}
\end{array}\right), \quad \boldsymbol{S}_{+}=\left(\begin{array}{c}
\mathbf{0} \\
\boldsymbol{p}_{0}
\end{array}\right), \quad \boldsymbol{S}_{-}=\left(\begin{array}{c}
\overline{\boldsymbol{s}}_{-} \\
\mathbf{0}
\end{array}\right) .
$$

Note that the stationary population equation (18) will be used in Sec. VI

\section{KCL NANOCLUSTER VACANCY DIFFUSION MODEL}

In this section, according to Ref. [30], we first present the vacancy diffusion model of $\mathrm{KCl}$ nanoclusters as an example of a practical problem, and then we introduce the corresponding Markov state model of the vacancy diffusion.

\section{A. Local minima and saddle points on the potential energy surface of a $\mathrm{KCl}$ nanocluster}

Let us assume that one chlorine ion is extracted from a cube of ionic crystal with equal $N_{L}$-atom edges and further that $N_{L}$ is an odd number $2 n_{L}+1$, and the resultant cluster with $N \equiv N_{L}{ }^{3}-1$ atoms is electrically neutral. We employ the two-body Coulomb plus Born-Mayer type potential model,

$$
v\left(r_{i j}\right)=\frac{Q_{i} Q_{j}}{4 \pi \epsilon_{0} r_{i j}}+A_{i j} \exp \left(\frac{R_{i}+R_{j}-r_{i j}}{\rho}\right),
$$

where $Q_{i}, Q_{j}$ are the charges of the $i$ th and $j$ th atoms, $\epsilon_{0}$ is the vacuum permittivity, and $r_{i j}$ is the distance between the $i$ th and $j$ th atoms. We use the values of the three parameters $A_{i j}, R_{i}$, and $\rho$ that were introduced by Tosi and Fumi in Ref. [63]: $A_{i j}=0.2210,0.2637$, and $0.1582 \mathrm{eV}$, respectively, for $\mathrm{K}-\mathrm{Cl}, \mathrm{K}-\mathrm{K}$, and $\mathrm{Cl}-\mathrm{Cl}$ pairs; $R_{i}=1.463$ and $1.585 \AA$ for $\mathrm{K}$ and $\mathrm{Cl}$, respectively; and $\rho=0.337 \AA$. Then, the total potential energy of the cluster is given by

$$
V\left(\boldsymbol{r}_{1}, \ldots, \boldsymbol{r}_{N}\right)=\sum_{i=1}^{N-1} \sum_{j=i+1}^{N} v\left(r_{i j}\right)
$$

In the course of the time evolution, the vacancy moves around the cluster, which introduces atomic mixing to the cluster. Note that the cubic form of the cluster is kept with the time evolution when the temperature is sufficiently low [29]. At such low temperatures, the position of the vacancy is specified by the cubic lattice point $\boldsymbol{n}=\left(n_{x}, n_{y}, n_{z}\right)$ with $-n_{L} \leqslant n_{x}, n_{y}, n_{z} \leqslant n_{L}$. Moreover, we are able to find the atomic structure corresponding to the vacancy lattice point $\boldsymbol{n}$ as follows: First, atoms are arranged at $d\left(m_{x}, m_{y}, m_{z}\right)$ with lattice constant $d=3.147 \AA$ for $\mathrm{KCl}$, where $\left(m_{x}, m_{y}, m_{z}\right) \neq \boldsymbol{n}$ and $-n_{L} \leqslant m_{x}, m_{y}, m_{z} \leqslant n_{L}$. Then, the configuration of the atoms is relaxed to the local minimum (LM) configuration, $\boldsymbol{r}=\left(\boldsymbol{r}_{1}, \ldots, \boldsymbol{r}_{N}\right)$, of the potential energy surface, e.g., by the conjugate gradient method [53]. In this way, $\boldsymbol{n}$ is assigned to the LM atomic structure as $\boldsymbol{r}_{\boldsymbol{n}}=\boldsymbol{r}$. We compute the LM configurations $\boldsymbol{r}_{\boldsymbol{n}}$ and the energies $V\left(\boldsymbol{r}_{\boldsymbol{n}}\right)$ for all $\boldsymbol{n}$. The LM datasets of $V\left(\boldsymbol{r}_{\boldsymbol{n}}\right)$ and $\boldsymbol{r}_{\boldsymbol{n}}$ are stored in a file in nondecreasing order of energy $V\left(\boldsymbol{r}_{\boldsymbol{n}}\right)$. For the sake of notational simplicity, the $i$ th lowest energy is denoted as $E_{i}$, and the corresponding LM, atomic configuration, and vacancy lattice point are denoted as $i$, $\boldsymbol{r}_{i}$, and $\boldsymbol{n}_{i}$, respectively. Then, we proceed to find out all of the saddle points ( $\mathrm{SPs}$ ) connecting the adjacent LMs, e.g., by the nudged elastic band method [32]. The corresponding saddle point connecting the $i$ th and $j$ th LMs, atomic configuration, and potential energy are denoted as $i j, \boldsymbol{r}_{i j}$, and $E_{i j}$, respectively. For the computational details of enumerating all of the LMs and SPs, we refer the reader to Ref. [30].

\section{B. Markov state model of $\mathrm{KCl}$ vacancy diffusion}

Let $f(\boldsymbol{r})$ denote the probability density function at a configuration $\boldsymbol{r}$. We suppose that the intra-LM relaxations are so fast that $f(\boldsymbol{r})$ is represented as

$$
f(\boldsymbol{r})=p_{1} f_{1}(\boldsymbol{r})+p_{2} f_{2}(\boldsymbol{r})+\cdots+p_{n} f_{n}(\boldsymbol{r}),
$$

where $n$ is the number of the LMs, $f_{i}(\boldsymbol{r})$ is the local equilibrium in the $i$ th-LM basin, and $p_{i}$ is the probability that $r$ is in the $i$ th-LM basin. We identify $p_{i}$ with the probability in the $i$ th state of the Markov state model. Then, the probability vector $\boldsymbol{P}$ of the Markov state model is given by $\boldsymbol{P}=\left(p_{1}, p_{2}, \ldots, p_{n}\right)^{T}$.

Next, we evaluate the transition rate $k_{i, j}$ from the $j$ th to the adjacent $i$ th state, when the potential barrier energies are sufficiently larger than the average kinetic energy of $k_{\mathrm{B}} T / 2$ for one degree of freedom at temperature $T$, where $k_{\mathrm{B}}$ denotes the Boltzmann constant. In this 
case, the transition rate $k_{i, j}$ from the $j$ th to the $i$ th state is given by

$$
k_{i, j}=\nu_{i, j} \exp \left(-\frac{E_{i j}-E_{j}}{k_{\mathrm{B}} T}\right) .
$$

Here, the prefactor $\nu_{i, j}$, called a frequency factor, is given by

$$
\nu_{i, j}=\frac{\prod_{k}^{\prime}\left(\boldsymbol{\nu}_{i}\right)_{k}}{\prod_{k}^{\prime}\left(\boldsymbol{\nu}_{i j}\right)_{k}}
$$

where $\boldsymbol{\nu}_{i}$ and $\boldsymbol{\nu}_{i j}$ are vibrational frequency vectors that are calculated from the Hessians at $\boldsymbol{r}_{i}$ and $\boldsymbol{r}_{i j}$, respectively. The product $\prod_{k}^{\prime}\left(\boldsymbol{\nu}_{*}\right)_{k}$ denotes the partial product of the positive frequency modes $\left(\boldsymbol{\nu}_{*}\right)_{k}>0$, where the imaginary frequency modes and the zero frequency modes are left out from the products.

Finally, the transition rate matrix $K$ is given by

$$
(K)_{i, j}=k_{i, j}(i \neq j) \quad \text { and } \quad(K)_{j, j}=-\sum_{i \neq j} k_{i, j},
$$

where the probability conservation equations $\sum_{i}(K)_{i, j}=$ 0 for $j=1,2, \ldots, n$ and the detailed balance conditions $(K)_{i, j}\left(\boldsymbol{P}_{\mathrm{eq}}\right)_{j}=(K)_{j, i}\left(\boldsymbol{P}_{\mathrm{eq}}\right)_{i}$ for $i, j=1,2, \ldots, n$ are satisfied since $\left(\boldsymbol{P}_{\text {eq }}\right)_{i} \propto \exp \left(-E_{i} / k_{\mathrm{B}} T\right)$.

In the following, we examine the slowest relaxation modes of the $\mathrm{KCl}$ vacancy diffusion model of $N_{L}=13$. To this end, we searched for the LMs and the SPs of the cluster, thereby finding $1099\left(=1+\left(N_{L}^{3}-1\right) / 2\right)$ LMs and 5472 SPs. Then, with the use of Eqs. (23), (24), and (25), we formed its rate matrix of $K$ at $k_{\mathrm{B}} T=0.03 \mathrm{eV}$, whose matrix dimension $n$ is 1099 and the number of nonzero offdiagonal elements is $10948(=5472 \times 2)$.

By diagonalizing $K$, we obtained the eigenvalues $\lambda_{i}$ and the corresponding relaxation modes $\boldsymbol{P}_{i}$ for $i=$ $0,1,2, \ldots, n-1$, where $\boldsymbol{P}_{0}=\boldsymbol{P}_{\text {eq }}$. In Sec. IV and Sec. V] we study the properties of the slowest relaxation $\boldsymbol{P}_{1}$ and the second slowest relaxations $\boldsymbol{P}_{2}, \boldsymbol{P}_{3}$, and $\boldsymbol{P}_{4}$ $\left(\lambda_{2}=\lambda_{3}=\lambda_{4}\right)$, respectively.

\section{THE SLOWEST RELAXATION MODE}

In this section, we show that the slowest relaxation mode of the $\mathrm{KCl}$ nanocluster makes effective use of the fast surface diffusion of the cluster, in terms of the mean first passage times, the free energy landscapes, and the atomic interactions.

\section{A. Dominant pathways}

Figure 1(a) shows the slowest relaxation mode of $\boldsymbol{P}_{1}$, from which we see that $\boldsymbol{P}_{1}$ has the probability excesses at around the origin $(0,0,0)$ and the probability shortages at around the eight vertices of $\left( \pm n_{L}, \pm n_{L}, \pm n_{L}\right)$. In
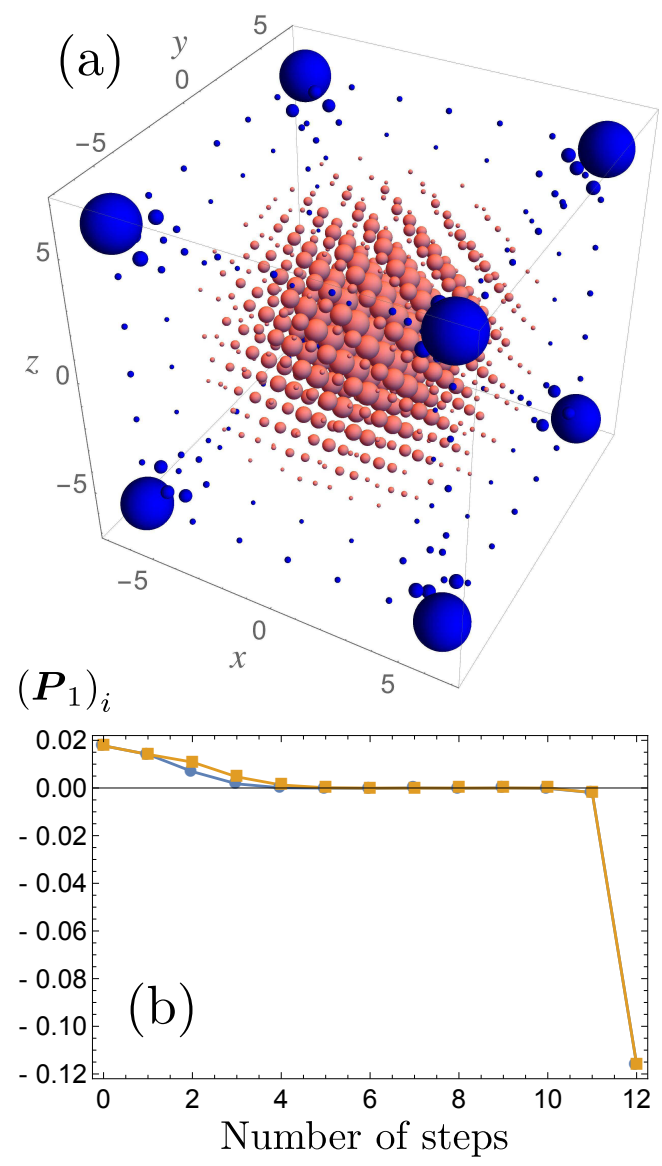

FIG. 1. The slowest relaxation mode, $\boldsymbol{P}_{1}$, at $k_{\mathrm{B}} T=0.03 \mathrm{eV}$ : (a) Red (light gray) and blue (dark gray) balls are depicted at vacancy lattice points of $\boldsymbol{n}_{i}$ with radii $\propto\left|\left(\boldsymbol{P}_{1}\right)_{i}\right|^{1 / 3}$ for $\left(\boldsymbol{P}_{1}\right)_{i}>0$ and $\left(\boldsymbol{P}_{1}\right)_{i}<0$, respectively. Note that $\boldsymbol{P}_{1}$ has cubic symmetry around the $x-, y$-, and $z$-axes. (b) $\left(\boldsymbol{P}_{1}\right)_{i}$ along pathways $\ell_{1}$ of Eq. (26) and $\ell_{2}$ of Eq. (27) are plotted as a function of the number of steps from the origin $(0,0,0)$ with blue circles connected by the lower line and orange squares connected by the upper line, respectively.

Fig. प(b), we also plot the values of $\left(\boldsymbol{P}_{1}\right)_{i}$ along two pathways from the origin to the vertex $\left(n_{L}, n_{L}, n_{L}\right)$, which clearly shows that they have the maximum values at the origin and positive values up to three steps from the origin and negative values at the vertices.

$\boldsymbol{P}_{1}$ decays with the rate of $\lambda_{1}=-1.92 \times 10^{5} \mathrm{~s}^{-1}$ over the course of time. Hence, the probabilistic flow from the center to the vertices is expected in the relaxation process of $\boldsymbol{P}_{1}$. To confirm this, we compute all of the probabilistic flows $f_{i, j}$ from $j$ to $i$, generated by $\boldsymbol{P}_{1}$, where $f_{i, j}$ is given by $f_{i, j}=k_{i, j} p_{j}-k_{j, i} p_{i}$ with $p_{i}=\left(\boldsymbol{P}_{1}\right)_{i}$ for $1 \leqslant i, j \leqslant n$. In Fig. 2, $f_{i, j}$ are represented by the arrows from $\boldsymbol{n}_{j}$ to $\boldsymbol{n}_{i}$ when $f_{i, j}>0$. We see that the probabilistic flows from the center to the vertices are generated. More precisely, the probabilistic flows are not uniform but mostly along the two dominant pathways of $\ell_{1}$ and $\ell_{2}$ as depicted in Fig. 2 . 


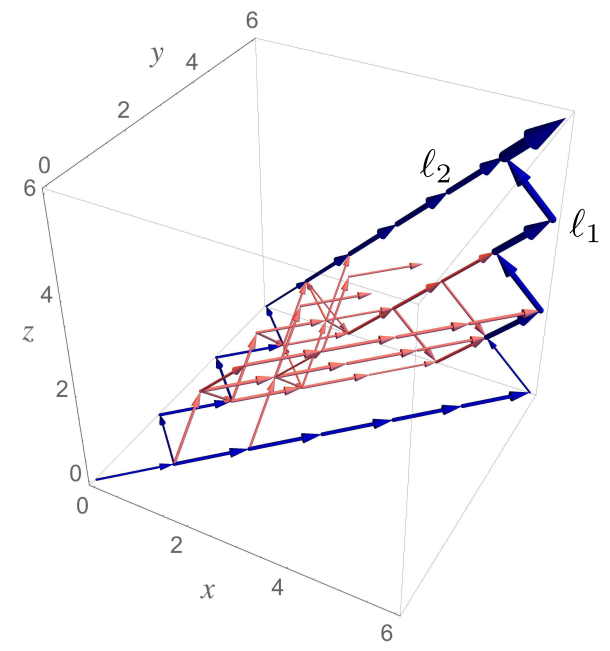

FIG. 2. Probabilistic flows $f_{i, j}$ of $\boldsymbol{P}_{1}$ at $k_{\mathrm{B}} T=0.03 \mathrm{eV}$ are represented by arrows from $\boldsymbol{n}_{j}$ to $\boldsymbol{n}_{i}$ with cylinder radii $\propto$ $\sqrt{\left|f_{i, j}\right|}$ for $f_{i, j}>0$. The flows have the same cubic symmetry as in Fig. 1 Hence, only the flows in a reduced zone $n_{y} \geqslant$ $n_{x} \geqslant n_{z} \geqslant 0$ are represented. We see that two pathways $\ell_{1}$ and $\ell_{2}$ in blue (dark gray) carry the dominant flows: $\ell_{1}$ is composed of the straight move from the origin $(0,0,0)$ to the edge center $(6,6,0)$ and the succeeding zigzag move to the vertex $(6,6,6) ; \ell_{2}$ is composed of the zigzag move from the origin to the face center $(0,6,0)$ and the succeeding straight move to the vertex. [See Eqs. (26) and (27).]

The dominant pathways of $\ell_{1}$ and $\ell_{2}$ are defined by the following algorithm that searches for the maximum flow pathways flowing into the terminals. First, we start with the terminal of the vertex $\left(n_{L}, n_{L}, n_{L}\right)=(6,6,6)$. The probabilistic flow from $(5,6,5)$ flows into the terminal $(6,6,6)$. The probabilistic flows from $(6,6,5)$ and $(4,6,4)$ flow into $(5,6,5)$, respectively. The dominant pathways via $(6,6,5)$ and $(4,6,4)$ are denoted as $\ell_{1}$ and $\ell_{2}$, respectively. We then search for the source flow of $\ell_{1}$ as follows. The probabilistic flow from $(5,6,4)$ is the maximum flow flowing into $(6,6,5)$. That from $(6,6,3)$ is the maximum flow flowing into $(5,6,4)$, and so on. This procedure continues until the source $(0,0,0)$ appears and gives the dominant pathway as

$$
\begin{aligned}
\ell_{1}= & (0,0,0) \rightarrow(1,1,0) \rightarrow \cdots \rightarrow(6,6,0) \\
& \rightarrow(5,6,1) \rightarrow(6,6,2) \rightarrow(5,6,3) \\
& \rightarrow(6,6,4) \rightarrow(5,6,5) \rightarrow(6,6,6) .
\end{aligned}
$$

The dominant paths of this kind are composed of six straight steps from the origin to the 12 centers of the edges $( \pm 6, \pm 6,0),( \pm 6,0, \pm 6),(0, \pm 6, \pm 6)$, followed by six zigzag steps from there to the vertices along the edges. Similarly, we search for the source flows flowing into
$(4,6,4)$ and obtain $\ell_{2}$ as

$$
\begin{aligned}
\ell_{2}= & (0,0,0) \rightarrow(1,1,0) \rightarrow(0,2,0) \rightarrow(1,3,0) \\
& \rightarrow(0,4,0) \rightarrow(1,5,0) \rightarrow(0,6,0) \rightarrow \\
& (1,6,1) \rightarrow \cdots \rightarrow(5,6,5) \rightarrow(6,6,6) .
\end{aligned}
$$

The dominant paths of the second kind are composed of six zigzag steps from the center to the six centers of the faces, $( \pm 6,0,0),(0, \pm 6,0),(0,0, \pm 6)$, followed by six straight steps from there to the vertices.

In other words, the dominant paths arriving at each vertex are the three $\ell_{1}$-type paths, which climb along the three edges connected to the vertex, and the three $\ell_{2}$-type paths, which move across the three faces containing the vertex. Note that these observations are consistent with our previous results from Ref. [42]. There, all states are divided into groups, called metabasins, that are located around the vertices, the edges, the faces, and the center part, and then the relaxation processes are described accurately by the renormalized transitions between these metabasins. That is to say, we have reconfirmed here that the essential pathways connecting the vertices, the edges, the faces, and the center part are indispensable for describing the slowest transport of probabilities.

\section{B. Mean first passage times}

Here, we consider why the dominant paths carrying large probabilistic flows are not almost straight, ninestep shortest paths from the origin to the vertices, such as $(0,0,0) \rightarrow(0,1,1) \rightarrow(1,1,2) \rightarrow(2,2,2) \rightarrow(2,3,3) \rightarrow$ $(2,4,4) \rightarrow(3,4,5) \rightarrow(4,5,5) \rightarrow(5,5,6) \rightarrow(6,6,6)$, but longer 12-step paths of $\ell_{1}$ and $\ell_{2}$ in Fig. 2. To this end, we examine the mean first passage times of $t_{j}$ from various initial states of $j$ to the sink states of the vertices $\left( \pm n_{L}, \pm n_{L}, \pm n_{L}\right)$.

Using Eq. (15), we compute $t_{j}$ for various initial states of $j$. The resulting $t_{j}$ are plotted in Fig. 3(a). We see that the states in the central part have large $t_{j}$ values, while the states on the surface have quite small values. That is, the $\mathrm{KCl}$ nanocluster is a hybrid system that combines entirely different microscopic diffusive regions: The central part is the region that is hard to move stochastically, whereas the surface part is the region that is quite easy to move. To see this more closely, we plot $t_{j}$ along the paths of $\ell_{1}$ and $\ell_{2}$ in Fig. 3(b), where both of $t_{j}$ have the maximum value at the origin and they are negligibly small compared to the maximum value in two layers from the surface.

Now, we see the reason why the detoured pathways are selected to be the dominant pathways, as depicted in Fig. 2. Namely, it is because all the dominant pathways prefer to pass the slow diffusion region of the central part as soon as possible, with the fewest steps of $n_{L}=6$, in order to make the most effective use of the fast diffusion in the surface region.

Next, we show that $\lambda_{1}$ can be evaluated approximately from $t_{j}$. The longest mean first passage time to the 

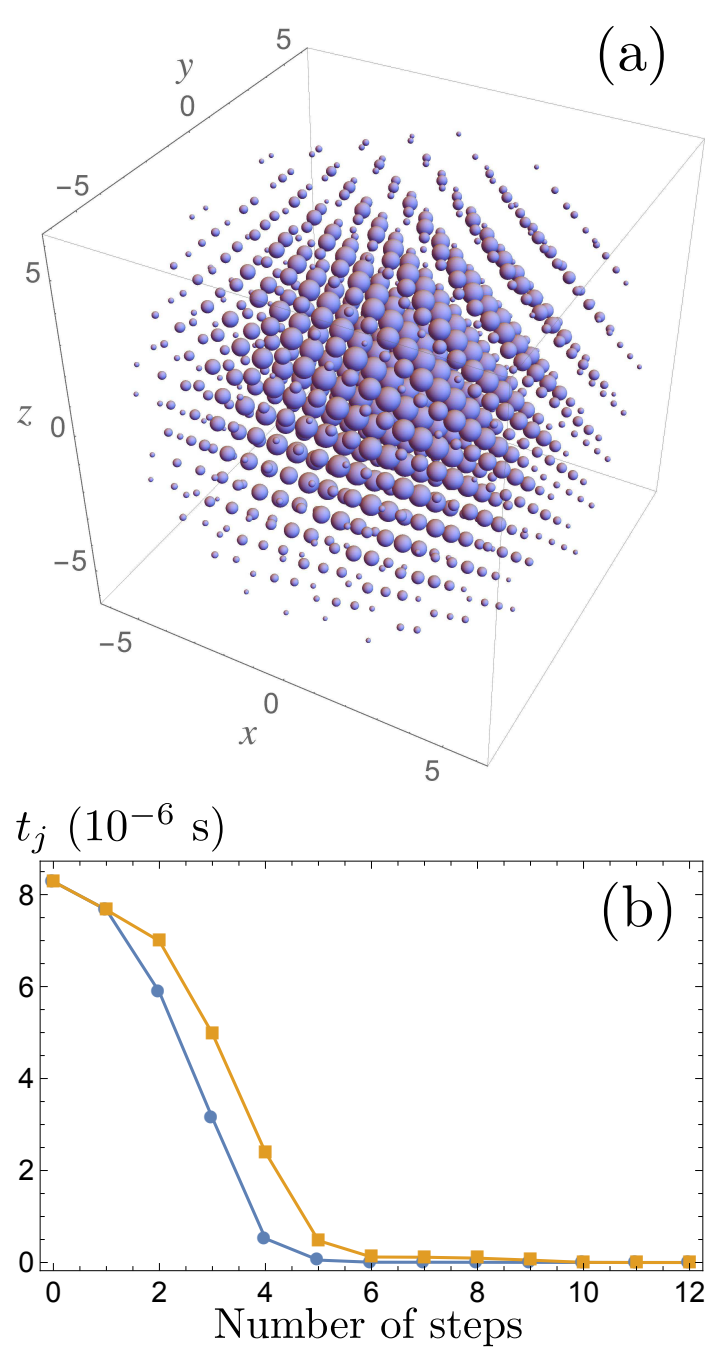

FIG. 3. Mean first passage times of $t_{j}$ at $k_{\mathrm{B}} T=0.03 \mathrm{eV}$, with perfect sinks connected to the vertices $( \pm 6, \pm 6, \pm 6)$ : (a) $t_{j}$ are represented by balls of radii $\propto\left|t_{j}\right|^{1 / 3}$ located at $\boldsymbol{n}_{j}$. The mean first passage times have cubic symmetry around the $x-, y$-, and $z$-axes. (b) $t_{j}$ along $\ell_{1}$ and $\ell_{2}$ are plotted as a function of the number of steps from the origin with blue circles connected by the lower line and orange squares connected by the upper line, respectively. Both of $t_{j}$ have the maximum value of $8.28 \times 10^{-6} \mathrm{~s}$ at the origin of $(0,0,0)$. The values of $t_{j}$ in the center part, where the number of steps is from 0 to 4 , have the same order of magnitude, while those in the two layers from the surface, where the number of steps is from 5 to 12 , are quite small values.

vertices is $t_{(0,0,0)}=8.28 \times 10^{-6} \mathrm{~s}$. The probability of being at the vertices in equilibrium is $\boldsymbol{P}_{\text {eq }}$ (vertices) $=$ $\sum_{i \in \text { vertices }}\left(\boldsymbol{P}_{\text {eq }}\right)_{i}=0.885$. We regard the equilibration time as the required time of constructing $\boldsymbol{P}_{\text {eq }}$ (vertices). Then, the equilibration time is approximately given by

$$
\boldsymbol{P}_{\text {eq }}(\text { vertices }) \times t_{(0,0,0)}=7.33 \times 10^{-6} \mathrm{~s} .
$$

The corresponding equilibration rate is given by the inverse of the equilibration time, $1.36 \times 10^{5} \mathrm{~s}^{-1}$. The

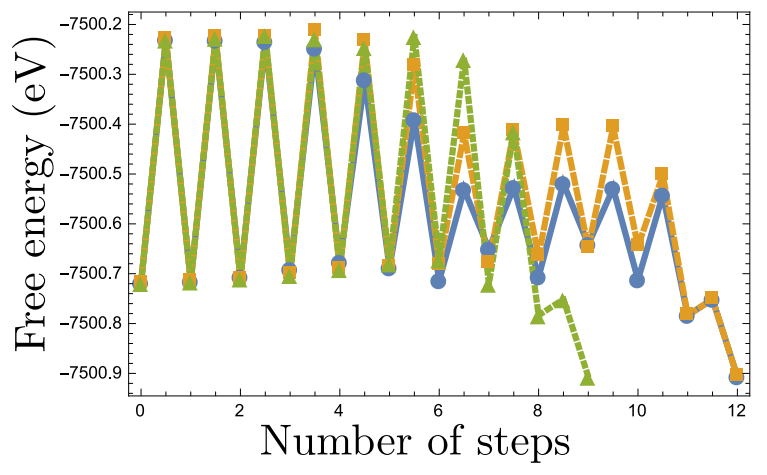

FIG. 4. Free energy sequences in units of $\mathrm{eV}$ are plotted at $k_{\mathrm{B}} T=0.03 \mathrm{eV}$, as functions of steps counted from the origin $(0,0,0)$ to a vertex along the geometric shortest path ( $\mathbf{\Lambda}$, green), along the dominant path, $\ell_{1}$, of Eq. (26) via an edge center ( $\bullet$, blue) and the dominant path, $\ell_{2}$, of Eq. (27) via a face center ( $\boldsymbol{\square}$, orange). The integer steps indicate the free energies of the local minima, and the half-integer steps indicate the free energies of the saddle points that connect the basins of adjacent local minima.

estimate agrees qualitatively with the values of $\left|\lambda_{1}\right|=$ $1.92 \times 10^{5} \mathrm{~s}^{-1}$, although it is a smaller value than $\left|\lambda_{1}\right|$.

This discrepancy arises because, although the actual excess probabilities in $\boldsymbol{P}_{1}$ are distributed in the central part as depicted in Fig. 1, the excess probability is approximated to the distribution concentrated on the origin, for the mean first passage time approximation of $\lambda_{1}$. We will revisit this point in Sec. VI]

\section{Free energy sequences}

Here, we consider the physical reason why the bottleneck of diffusion in the Markov state model of the $\mathrm{KCl}$ nanocluster is located at the central part. To this end, we examine the following free energies for the LMs of $j$ and the SPs of $i j$, respectively:

$$
\begin{gathered}
F_{j}=E_{j}-k_{\mathrm{B}} T \ln \prod_{k}^{\prime}\left(\boldsymbol{\nu}_{j}\right)_{k}, \\
F_{i j}=E_{i j}-k_{\mathrm{B}} T \ln \prod_{k}^{\prime}\left(\boldsymbol{\nu}_{i j}\right)_{k},
\end{gathered}
$$

where $k_{i, j}=\exp \left[-\beta\left(F_{i j}-F_{j}\right)\right]$ holds. Then, the free energy sequence of local minima and saddle points along a pathway $i_{0} \rightarrow i_{1} \rightarrow \cdots \rightarrow i_{s}$ is given by $F_{i_{0}}, F_{i_{0} i_{1}}, F_{i_{1}}, F_{i_{1} i_{2}}, F_{i_{2}}, \ldots F_{i_{s}}$.

In Fig. [4 we plot the free energy sequences of the dominant pathways of $\ell_{1}$ [Eq. [26)] and $\ell_{2}$ [Eq. (27)]. Along these dominant pathways, the activation energies for the inner transitions $i_{k} \rightarrow i_{k+1}$ are about $\Delta F_{i_{k+1}, i_{k}}=$ $F_{i_{k} i_{k+1}}-F_{i_{k}} \approx 0.5 \mathrm{eV}$, and hence the transition rates become quite low rates of $k_{i_{k+1}, i_{k}} \approx 6 \times 10^{5} \mathrm{~s}^{-1}$ at $k_{\mathrm{B}} T=0.03 \mathrm{eV}$. In contrast, those for the surface transitions are $\Delta F_{i_{k+1}, i_{k}} \approx 0.2 \mathrm{eV}$, and the transition rates are 
about $k_{i_{k+1}, i_{k}} \approx 1 \times 10^{10} \mathrm{~s}^{-1}$, which are about $10^{4}$ times higher than the inner rates, at the same temperature.

For comparison, we also plot the free energy sequence along the nine-step geometric shortest path in Fig. [4 We see that the first seven steps are in the slow diffusion region and the last two steps are in the faster surface diffusion region. Therefore, the geometric shortest path cannot be dominant, because the extra steps in the slower diffusion region reduce its diffusive flow drastically.

Note that the activation free energies of $\Delta F_{i_{k}, i_{k+1}} \gtrsim$ $0.2 \mathrm{eV}$ are sufficiently larger than $k_{\mathrm{B}} T=0.03 \mathrm{eV}$ and hence the harmonic approximation (23) used in this study is accurate.

\section{Activation energies on potential energy landscapes}

Next, we show that it can be understood in terms of the interatomic interaction energies why the surface activation free energies are so small compared to the inner ones.

First, from Eqs. (24), (29), and (30), $\Delta F_{i, j}=E_{i j}-$ $E_{j}+k_{\mathrm{B}} T \ln \nu_{i, j}$ holds. At the low temperature of $k_{\mathrm{B}} T=$ $0.03 \mathrm{eV}, k_{\mathrm{B}} T \ln \nu_{i, j} \approx 0.03 \mathrm{eV}$ is negligible compared to $\Delta F_{i, j} \approx 0.5 \mathrm{eV}$, and $\Delta F_{i, j} \approx E_{i j}-E_{j}$ holds. Hence, in the following we consider the activation energies, $\Delta E_{i, j}=$ $E_{i j}-E_{j}$, of various transitions.

We examine the activation energy of $\Delta E_{i, j}$ when the vacancy lattice points $\boldsymbol{n}_{i}$ and $\boldsymbol{n}_{j}$ are present in the inner part of the cluster. In this case, $E_{i}$ and $E_{j}$ are almost the same, as shown in Fig. 4 , and hence $\Delta E_{i, j}$ is determined by the energy increase from $E_{i} \simeq E_{j}$, due to the deformation of the crystal structure near the lattice defect. To quantify the deformation energies, we introduce the individual potential energy of the $k$ th atom as

$$
V_{k}=\frac{1}{2} \sum_{l \neq k} v\left(r_{k l}\right)
$$

Then, the total potential energy of Eq. (21) is represented as

$$
V(\boldsymbol{r})=\sum_{k} V_{k}
$$

Note that $V_{k}$ is half of the required energy to remove the $k$ th atom from the cluster, since $V\left(\boldsymbol{r}_{1}, \ldots, \boldsymbol{r}_{k}, \ldots \boldsymbol{r}_{N}\right)-$ $V\left(\boldsymbol{r}_{1}, \ldots, \boldsymbol{r}_{k-1}, \boldsymbol{r}_{k+1} \ldots, \boldsymbol{r}_{N}\right)=2 V_{k}$ holds. Figures [5(a) and 5 (c), respectively, show the values of $V_{k}$ for the LMs of the vacancy lattice points of $\boldsymbol{n}_{1}=(0,0,0)$ and $\boldsymbol{n}_{2}=(1,1,0)$. We see that the changes of $V_{k}$ are concentrated in the vicinities of the vacancies at around $\boldsymbol{r}_{1}=d \boldsymbol{n}_{1}$ and $\boldsymbol{r}_{2}=d \boldsymbol{n}_{2}$ with lattice constant $d$, and that $V_{k}$ of $\mathrm{Cl}$ and $\mathrm{K}$ atoms decrease and increase, respectively, when approaching the vacancy positions. Figure 5 (b) shows the values of $V_{k}$ for the SP connecting the LMs of $\boldsymbol{n}_{1}$ and $\boldsymbol{n}_{2}$. The SP has the high energy $\mathrm{Cl}$ atom as the lattice defect at around the midpoint,
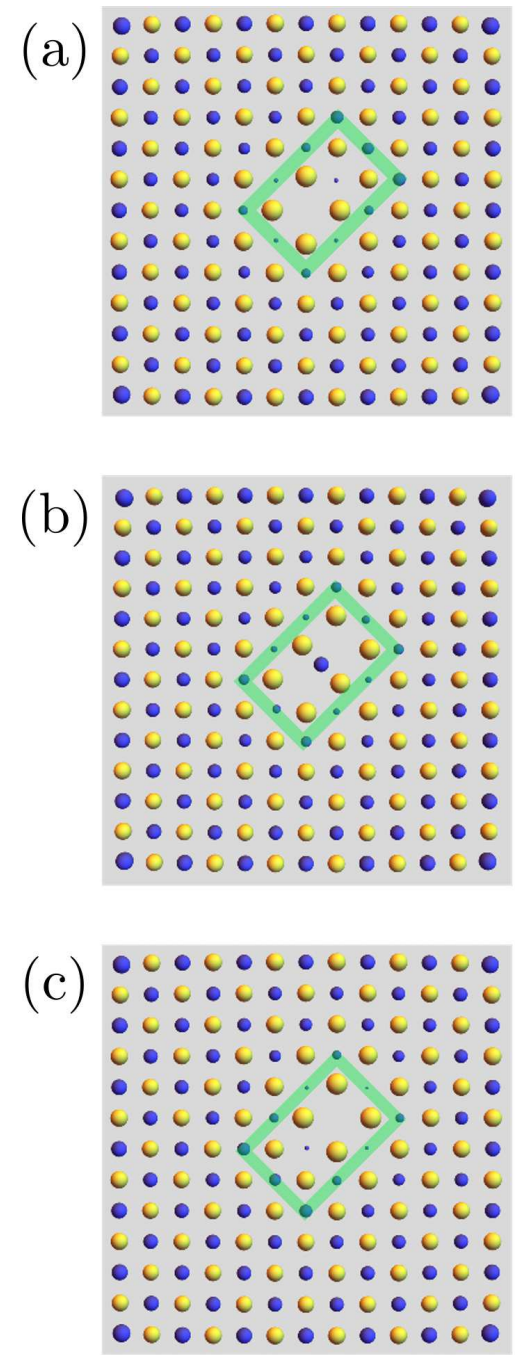

FIG. 5. Individual energies $V_{k}$ of $k$ th atoms contained in the $z=0$ planes are shown for (a) the LM of the vacancy lattice point of $\boldsymbol{n}_{1}=(0,0,0)$, (b) the SP connecting LMs of $\boldsymbol{n}_{1}$ and $\boldsymbol{n}_{2}=(1,1,0)$, and (c) the LM of the vacancy lattice point of $\boldsymbol{n}_{2}$. The $\mathrm{Cl}$ and $\mathrm{K}$ atoms of individual energies $V_{k}$ are represented by blue (dark gray) and yellow (light gray) balls with radii $\propto \sqrt[3]{V_{k}-\min _{k}\left\{V_{k}\right\}}$. The defect neighbors are defined by the regions inside the green (gray) frames of $|x-y|<1.2 d,-1.2 d<x+y<3.2 d$, and $|z|<2.2$. (See the text.)

$\boldsymbol{r}_{1,2}=d(1 / 2,1 / 2,0)$, of the vacancy positions. In this case, too, $V_{k}$ of $\mathrm{Cl}$ and $\mathrm{K}$ atoms decrease and increase, respectively, when approaching the defect of the highenergy $\mathrm{Cl}$ atom.

Next, we show that $\Delta E_{i, j}$ can be estimated with use of the local $V_{k}$ values around the defects. To this end, we obtain the local energies $E_{1}^{\text {loc. }}=-134.34 \mathrm{eV}$, $E_{1,2}^{\text {loc. }}=-133.804 \mathrm{eV}$, and $E_{2}^{\text {loc. }}=-134.334 \mathrm{eV}$, which are the sums of $V_{k}$ inside the local regions surrounded by the green (gray) rectangle frames in Figs. 圀(a), 囵(b), and 5(c), respectively. Hence, the activation energies eval- 
uated from these local energies are given by $\Delta E_{2,1}^{\text {loc. }}=$ $E_{1,2}^{\text {loc. }}-E_{1}^{\text {loc. }}=0.530 \mathrm{eV}$ and $\Delta E_{1,2}^{\text {loc. }}=E_{1,2}^{\text {loc. }}-E_{2}^{\text {loc. }}=$ $0.536 \mathrm{eV}$, which agree qualitatively with the exact activation energies of $\Delta E_{2,1}=0.58 \mathrm{eV}$ and $\Delta E_{1,2}=0.58$ $\mathrm{eV}$.

Similarly, we also evaluate the local activation energies of other types of activation processes as listed in Table 【 We see that the other types of activation energies are also described suitably by the local activation energies. Therefore, we have confirmed that all of the activation energies can be interpreted as the energy rises due to the local lattice deformations generated around the lattice defects.

Also, the local deformation assumption leads to the approximate relations of $\Delta E_{\text {face } \leftarrow \text { face }} \approx \Delta E_{\text {inner } \leftarrow \text { inner }} / 2$ and $\Delta E_{\text {edge } \leftarrow \text { face }} \approx \Delta E_{\text {inner } \leftarrow \text { inner }} / 4$, which are implied in Table II To understand these relations, we assume for simplicity that the deformation energy is uniformly distributed inside the ball of radius $a \approx d$ located at the defect point. Assuming further that the deformation energy per unit volume is given by $\epsilon$, then the activation energies for inner vacancies are estimated as $\Delta E_{\text {inner } \leftarrow \text { inner }}=4 \pi a^{3} \epsilon / 3(=0.58 \mathrm{eV})$.

Next, we consider the activation energies for vacancies in a face. In this case, the energies of adjacent local minima are also supposed to be the same for simplicity. Since the deformed regions are half of the inner case, the deformation energies of the vacancies in the faces are estimated to be $\Delta E_{\text {face } \leftarrow \text { face }}=4 \pi a^{3} \epsilon / 3 / 2=$ $\Delta E_{\text {inner } \leftarrow \text { inner }} / 2(=0.29 \mathrm{eV})$.

Moreover, when a vacancy inside a face moves to an adjacent edge, the deformation energy reduces to half of $\Delta E_{\text {face } \leftarrow \text { face }}$, since the deformed region is halved from that of the transition in a face. Hence, we have $\Delta E_{\text {edge } \leftarrow \text { face }} \approx \Delta E_{\text {inner } \leftarrow \text { inner }} / 4(=0.15 \mathrm{eV})$. We see that the estimated values agree quantitatively with the exact values. Lastly, when the vacancy in a face moves to an adjacent vertex, the deformation energy is evaluated to be halved to $\Delta E_{\text {edge } \leftarrow \text { face }}$. Thus, we have the following approximation: $\Delta E_{\text {vertex } \leftarrow \text { face }} \approx \Delta E_{\text {inner } \leftarrow \text { inner }} / 8(=$ $0.07 \mathrm{eV}$ ), which agrees qualitatively with the exact value of $0.027 \mathrm{eV}$.

Here, we have revealed that the activation energies for the system of $n_{L}=6$ are determined by the local deformation energies of $\Delta E_{i, j}^{\text {loc. }}$ around the defects. Accordingly, the activation energies are supposed to be almost independent of the system size of $n_{L}$. In fact, we have $\Delta E_{\text {inner } \leftarrow \text { inner }}=\Delta E_{(0,1,1) \leftarrow(0,0,0)}=$ $0.58 \mathrm{eV}, \Delta E_{\text {face } \leftarrow \text { face }}=\Delta E_{(4,1,1) \leftarrow(4,0,0)}=0.33 \mathrm{eV}$, $\Delta E_{\text {edge } \leftarrow \text { face }}=\Delta E_{(4,4,0) \leftarrow(4,3,1)}=0.16 \mathrm{eV}$, and $\Delta E_{\text {vertex } \leftarrow \text { face }}=\Delta E_{(4,4,4) \leftarrow(4,3,3)}=0.03 \mathrm{eV}$ for $n_{L}=4$. These results show that all types of activation energies are indeed almost independent of the system size when $n_{L} \geqslant 4$.

On the other hand, for $n_{L}=2$, we have $\Delta E_{\text {inner } \leftarrow \text { inner }}=\Delta E_{(0,1,1) \leftarrow(0,0,0)}=0.47 \mathrm{eV}$, $\Delta E_{\text {face } \leftarrow \text { face }}=\Delta E_{(2,1,1) \leftarrow(2,0,0)}=0.39 \mathrm{eV}$, $\Delta E_{\text {edge } \leftarrow \text { face }}=\Delta E_{(2,2,0) \leftarrow(2,1,1)}=0.3 \mathrm{eV}$, and
$\Delta E_{\text {vertex } \leftarrow \text { face }}=\Delta E_{(2,2,2) \leftarrow(2,1,1)}=0.05 \mathrm{eV}$, which shows that the uniform local deformation assumption for the activation energies employed above does not hold for $n_{L}=2$. In other words, the cluster of $n_{L}=2$ is too small to separate the deformations of the surface from those of the central portion, and thus some non-negligible couplings are generated between the inner and surface deformations. As a result of the couplings, the relatively high activation energies between inner transitions are decreased, while the other relatively low activation energies between surface transitions are increased for $n_{L}=2$.

In addition, the saddle connectivity graphs of $n_{L}=4$, 6 , and 8 depicted in Ref. [30] also show visually that the activation energies of $\Delta E_{\text {inner } \leftarrow \text { inner }}, \Delta E_{\text {face } \leftarrow \text { face }}$, $\Delta E_{\text {edge } \leftarrow \text { face }}$, and $\Delta E_{\text {vertex } \leftarrow \text { face }}$ are almost independent of the sizes $n_{L}$ of the clusters.

\section{THE SECOND SLOWEST RELAXATIONS}

In this section, we examine the second slowest relaxations of $\lambda_{2}, \lambda_{3}, \lambda_{4}=-3.89 \times 10^{5} \mathrm{~s}^{-1}$.

In Fig. 6. we plot $\boldsymbol{P}_{2}$ in the same way as in Fig. 1. The probability deviations of $\boldsymbol{P}_{2}$ are polarized in the $x$ direction. Here, the probability excess is in the region of $x>0$, the probability shortage is in $x<0$, and the probabilities are zero in $x=0$. From this observation, the relaxation process is expected as follows: the probability excess moves in the opposite $x$-direction, the probability shortage moves in the $x$-direction, and these pairs meet

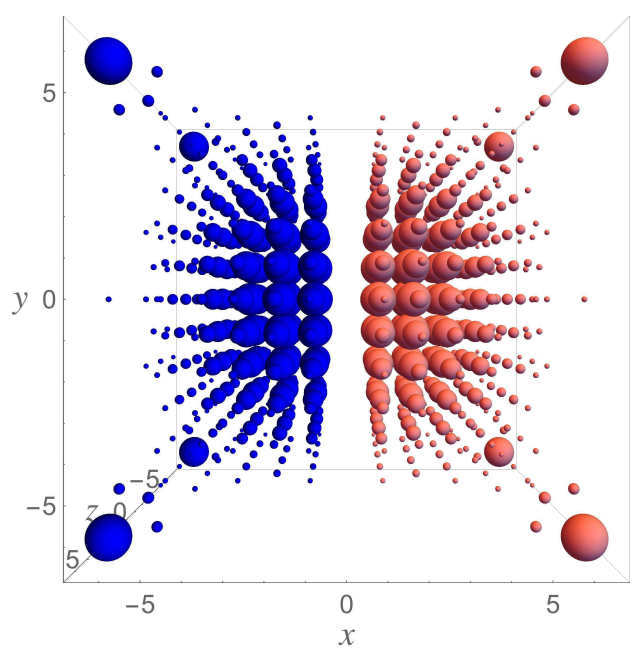

FIG. 6. We plot the second slowest relaxation mode of $\boldsymbol{P}_{2}$ in the same manner as in Fig. 1. $\boldsymbol{P}_{2}$ has the four-fold rotational symmetry around the $x$-axis. The relaxation mode is polarized in the $x$-direction, where the excess and shortage of probability are distributed, respectively, in $x>0$ and $x<0$, symmetrically with respect to the plane of $x=0$. Similarly to $\boldsymbol{P}_{2}, \boldsymbol{P}_{3}$ and $\boldsymbol{P}_{4}$ are the relaxation modes, which are polarized in the $y$ - and $z$-directions, respectively. 
TABLE I. Activation energies $\Delta E_{i, j}$ and local activation energies $\Delta E_{i, j}^{\text {loc. }}$ of vacancy transitions in the KCl cluster of $n_{L}=6$ are enumerated in units of eV. $\Delta E_{1,2}=E_{1,2}-E_{2}$ and $\Delta E_{1,2}^{\text {loc. }}=E_{1,2}^{\text {loc. }}-E_{2}^{\text {loc. }}$, where $E_{1,2}^{\text {loc. }}$ and $E_{2}^{\text {loc. }}$ are sums of individual atomic energies around the defect points. (See the text.)

\begin{tabular}{cccc}
\hline Activation type & $\boldsymbol{n}_{1} \leftarrow \boldsymbol{n}_{2}$ & $\Delta E_{1,2}$ & $\Delta E_{1,2}^{\text {loc. }}$ \\
\hline \hline Inner $\leftarrow$ Inner & $(1,1,0) \leftarrow(0,0,0)$ & 0.58 & 0.53 \\
Face $\leftarrow$ Face & $(6,1,1) \leftarrow(6,0,0)$ & 0.32 & 0.33 \\
Edge $\leftarrow$ Face & $(6,6,0) \leftarrow(6,5,1)$ & 0.15 & 0.18 \\
Vertex $\leftarrow$ Face & $(6,6,6) \leftarrow(6,5,5)$ & 0.027 & 0.036 \\
\hline
\end{tabular}

with each other in the region of $x=0$, to be annihilated.

To confirm this expectation, we evaluated the mean first passage times with sinks connected to $\left(0, n_{y}, n_{z}\right)$ for $-n_{L} \leqslant n_{y}, n_{z} \leqslant n_{L}$. The longest passage time is $t_{(2,0,0)}=4.05 \times 10^{-6} \mathrm{~s}$. The resulting rate of this process is $2.47 \times 10^{5} \mathrm{~s}^{-1}$. Also here, the estimated values of the rate agree qualitatively with $\left|\lambda_{2}\right|$, but they are somewhat smaller than the exact rate of $\left|\lambda_{2}\right|=3.89 \times 10^{5} \mathrm{~s}^{-1}$, because this approximate rate is evaluated only from the longest mean first passage time, as discussed in Sec.IVB

Finally, we show that the approximate relation of $\lambda_{2} \approx 2 \lambda_{1}$ holds. Here, the value of $\lambda_{2}$ evaluated from the mean first passage time from $(2,0,0)$ to $x=0$ is approximated by that from $(2,0,0)$ to $(0,0,0)$. From Fig. 1(b), we see that $\lambda_{1}$ is approximately evaluated from the mean first passage time from $(0,0,0)$ to $(4,0,0)$, which is approximately twice as long as that from $(0,0,0)$ to $(2,0,0)$ since each of the transitions requires almost the same

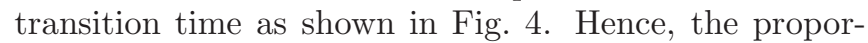
tional relation $1 /\left|\lambda_{1}\right|: 1 /\left|\lambda_{2}\right|=4: 2$ holds, and thus $\lambda_{2}=2 \lambda_{1}$ holds. Similarly, we can derive the approximate relations of $\lambda_{3}=2 \lambda_{1}$ and $\lambda_{4}=2 \lambda_{1}$.

In this section, we have confirmed that the second slowest relaxations of $\boldsymbol{P}_{2}, \boldsymbol{P}_{3}$, and $\boldsymbol{P}_{4}$, respectively, smooth out the nonequilibrium distribution deviations in the $x$-, $y$-, and $z$-directions in the course of time, and the bottleneck processes for the second slowest relaxations are also the slow diffusions inside the cluster. This fact allows us to derive the approximate relation of $\lambda_{2}, \lambda_{3}, \lambda_{4} \approx 2 \lambda_{1}$.

\section{SYMMETRIC EVALUATION OF RELAXATION RATES}

\section{A. Symmetric population method}

In the previous sections, we have successfully evaluated the values of the slowest and second slowest relaxation rates with the use of the mean first passage times. Recall that the usages of the mean first passage times for the slowest and second slowest relaxations were different. That is, for the slowest relaxation, the mean first passage times concerning particles were used, whereas, for the second slowest relaxations, the mean annihilation times of particle-hole pairs were evaluated with the uses of the mean first passage times.
The difference manifests itself in the symmetry of relaxation times under exchanging the sinks and sources. Namely, the approaches to evaluating the slowest and second slowest relaxation rates, respectively, give the relaxation times that are asymmetric and symmetric for exchanging sinks and sources. In fact, as shown in Eq. (28), the slowest relaxation time is $7.33 \times 10^{-6} \mathrm{~s}$ with the source and the sinks being connected to the origin and the vertices, respectively, whereas, with the sources and the sink being connected to the vertices and the origin, the mean first passage time is given by $\tau^{\prime}=3.82 \times 10^{-3} \mathrm{~s}$ and thus the relaxation time is $\left(\boldsymbol{P}_{\text {eq }}\right)_{(0,0,0)} \times \tau^{\prime}=8.24 \times 10^{-3} \mathrm{~s}$. The symmetry corresponds to the property that $\boldsymbol{P}_{i}$ and $-\boldsymbol{P}_{i}$ have the same value of $\lambda_{i}$, and hence it is required for a consistent treatment.

Here, we develop an alternative population method for estimating mean first passage times that is symmetric under the exchange of the sinks and sources.

To this end, we consider the following stationary population equation:

$$
\begin{gathered}
K \boldsymbol{Q}+\boldsymbol{S}=\mathbf{0} \\
\text { with }\left\|\boldsymbol{Q}_{+}\right\|=\left\|\boldsymbol{Q}_{-}\right\|, \quad\left\|\boldsymbol{S}_{+}\right\|=\left\|\boldsymbol{S}_{-}\right\|=1
\end{gathered}
$$

Here, $\boldsymbol{Q}=\boldsymbol{Q}_{+}-\boldsymbol{Q}_{-}$and $\boldsymbol{S}=\boldsymbol{S}_{+}-\boldsymbol{S}_{-}$, where the positive population $\boldsymbol{Q}_{+}$and the negative population $-\boldsymbol{Q}_{-}$ are given by

$$
Q_{+}=\frac{Q+|Q|}{2}, \quad Q_{-}=-\frac{Q-|Q|}{2},
$$

with $|\boldsymbol{Q}| \equiv\left(\left|q_{1}\right|,\left|q_{2}\right|, \ldots,\left|q_{n}\right|\right)$. The source part $\boldsymbol{S}_{+}$and the sink part $-\boldsymbol{S}_{-}$are, respectively, given by

$$
\boldsymbol{S}_{+}=\frac{\boldsymbol{S}+|\boldsymbol{S}|}{2}, \quad \boldsymbol{S}_{-}=-\frac{\boldsymbol{S}-|\boldsymbol{S}|}{2}
$$

with $|\boldsymbol{S}| \equiv\left(\left|s_{1}\right|,\left|s_{2}\right|, \ldots,\left|s_{n}\right|\right)$. With use of the stationary solution $\overline{\boldsymbol{P}}$, satisfying Eqs. (18) and (19), the stationary solution $\boldsymbol{Q}$ of Eqs. (33) and (34) is given by

$$
\boldsymbol{Q}=\overline{\boldsymbol{P}}-\|\overline{\boldsymbol{P}}\| \boldsymbol{P}_{\text {eq }},
$$

In fact, $Q$ given in Eq. (37) satisfies the constraint $\left\|Q_{+}\right\|=\left\|Q_{-}\right\|$, because $\left\|Q_{+}\right\|-\left\|Q_{-}\right\|=\|Q\|=$ $\left\|\left(\overline{\boldsymbol{P}}-\|\overline{\boldsymbol{P}}\| \boldsymbol{P}_{\text {eq }}\right)\right\|=\|\overline{\boldsymbol{P}}\|-\|\overline{\boldsymbol{P}}\|=0$ holds.

As illustrated in Fig. Z (b), the negative population $-\boldsymbol{Q}_{-}$can be interpreted as the hole population of $\boldsymbol{Q}_{-}$. 

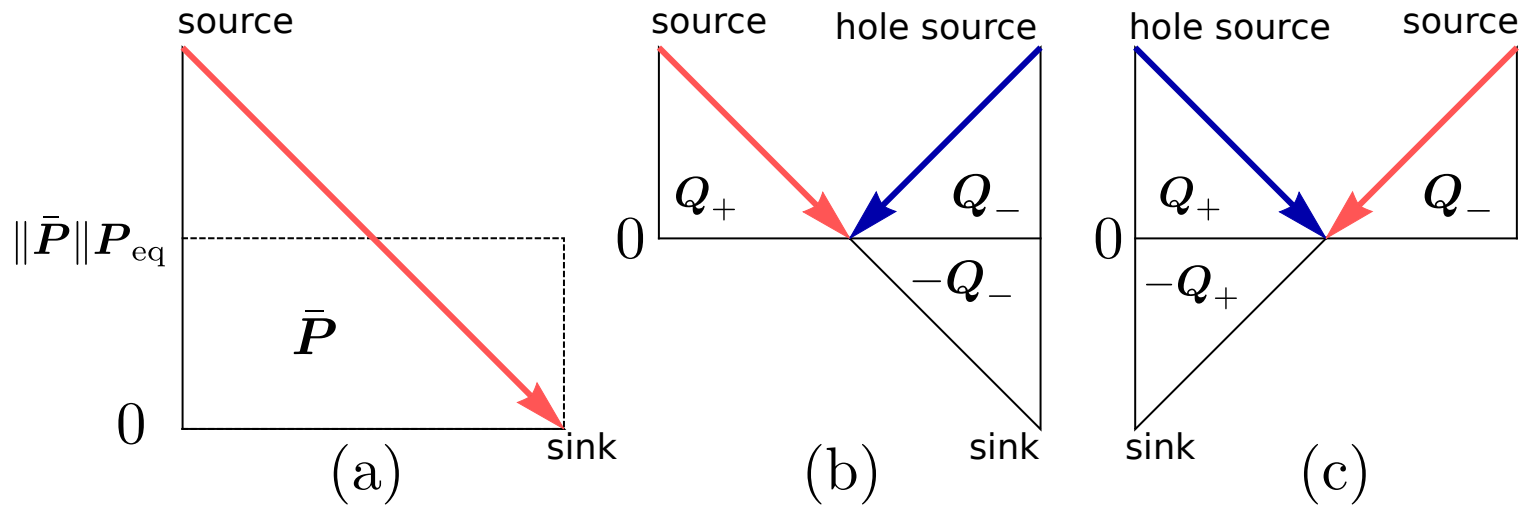

FIG. 7. Schematic illustration of the population methods. (a) The stationary particle population of $(\overline{\boldsymbol{P}})_{i}$ satisfying Eqs. (18) and (19) is shown as a function of states $i$. The red (light gray) arrow indicates how the particles injected at the source move diffusively to the sink. We also show $\|\overline{\boldsymbol{P}}\| \boldsymbol{P}_{\text {eq }}$ with a dashed line, to illustrate the latter term in Eq. (37). (b) The symmetric stationary population $\boldsymbol{Q}$ with a source and a sink connected to the left and right end states, respectively, is given by Eq. (37). The negative population $-\boldsymbol{Q}_{-}$is interpreted as the stationary population $\boldsymbol{Q}_{-}$of holes. The blue (gray) arrow indicates how the holes injected at the hole source move diffusively. Particles from the source and the holes meet at the center and are annihilated by pair annihilation. (c) The stationary population under the exchange of the sink and source is shown. In this case, particles of population $\boldsymbol{Q}_{-}$move to the left and holes of population $\boldsymbol{Q}_{+}$move to the right. The particles and the holes meet at the center to be annihilated. Both of the mean annihilation times in (b) and (c) agree with the mean first passage time, $\left\|\boldsymbol{Q}_{+}\right\|=\left\|\boldsymbol{Q}_{-}\right\|$, of particles and holes. (See the text.)

Hence, the mean annihilation times of particles and holes are, respectively, given by the first passage times of $\left\|\boldsymbol{Q}_{+}\right\|$ and $\left\|\boldsymbol{Q}_{-}\right\|$as discussed in Sec. $\mathbf{V}$ Similarly, $\boldsymbol{S}_{-}$is interpreted as a hole source part that adds one hole per unit time. Hence, the constraint of $\left\|\boldsymbol{S}_{+}\right\|=\left\|\boldsymbol{S}_{-}\right\|=1$ means that $\boldsymbol{S}_{+}$adds one particle per unit time, and $\boldsymbol{S}_{-}$adds one hole per unit time. Note that, since $\boldsymbol{P}_{\text {eq }}$ satisfies the detailed balance condition, the particle flows of $\overline{\boldsymbol{P}}$ and $\boldsymbol{Q}$ are the same, and so are their dominant pathways, as illustrated in Figs. 7(a) and 7(b).

The constraint $\left\|\boldsymbol{Q}_{+}\right\|=\left\|\boldsymbol{Q}_{-}\right\|$means that the mean first passage times are symmetric under exchanging the sinks and sources. In fact, by exchanging the sinks and sources, $\boldsymbol{S}$ and $\boldsymbol{Q}$ are, respectively, converted to $-\boldsymbol{S}$ and $-\boldsymbol{Q}$, and hence $\boldsymbol{Q}_{+}$and $\boldsymbol{Q}_{-}$are, respectively, converted to $\boldsymbol{Q}_{-}$and $\boldsymbol{Q}_{+}$, as illustrated in Fig. Z(c). Hence, the mean first passage times of Eqs. (33) and (34) satisfy $\left\|Q_{-}\right\|=\left\|Q_{+}\right\|$with sinks and sources exchanged, which is the same value as the value before the exchange.

Here, with this symmetric population method, we evaluate the slowest relaxation rate of $\lambda_{1}$ for the vacancy diffusion model of the $\mathrm{KCl}$ nanocluster. Setting

$$
\left(\boldsymbol{S}_{+}\right)_{(0,0,0)}=1,\left(\boldsymbol{S}_{-}\right)_{\left( \pm n_{L}, \pm n_{L}, \pm n_{L}\right)}=1 / 8
$$

and otherwise $\left(\boldsymbol{S}_{ \pm}\right)_{i, j, k}=0$, we evaluated the symmetric stationary solution of Eq. (41), thereby obtaining the slowest relaxation time of $\left\|\boldsymbol{Q}_{+}\right\|=\left\|\boldsymbol{Q}_{-}\right\|=7.92 \times 10^{-6}$ s. Namely, with this symmetric method, we obtained an approximation of the exact slowest relaxation time of $-1 / \lambda_{1}=5.2 \times 10^{-6} \mathrm{~s}$, which is symmetric under exchanging the sinks and sources and as accurate as the asymmetric result of $7.33 \times 10^{-6} \mathrm{~s}$ given in Sec. IV]
For the second slowest relaxation, we set

$$
\left(\boldsymbol{S}_{+}\right)_{(2,0,0)}=1,\left(\boldsymbol{S}_{-}\right)_{(-2,0,0)}=-1
$$

and otherwise $\left(\boldsymbol{S}_{ \pm}\right)_{i, j, k}=0$, thereby obtaining the symmetric result of $\left\|\boldsymbol{Q}_{+}\right\|=\left\|\boldsymbol{Q}_{-}\right\|=4.05 \times 10^{-6}$ s, which of course agrees with the result given in Sec. $\mathrm{V}$

In this subsection, we have developed the symmetric population method for mean first passage times, which enables us to approximately evaluate the slowest relaxation times symmetrically by exchanging the sinks and sources.

\section{B. Symmetric population method as an inverse power method}

Here, we show that the iterative use of the symmetric population method enables us to compute the slowest relaxation times accurately.

First, the $n \times n$ matrix $P=\left(\boldsymbol{P}_{\text {eq }}, \boldsymbol{P}_{1}, \boldsymbol{P}_{2}, \ldots, \boldsymbol{P}_{n-1}\right)$ is invertible, where $\boldsymbol{P}_{i}$ is the eigenvector corresponding to the $i$ th relaxation mode. We expand $\boldsymbol{S}$ and $\boldsymbol{Q}$ as

$$
\begin{aligned}
& \boldsymbol{S}=s_{0}^{\prime} \boldsymbol{P}_{\text {eq }}+s_{1}^{\prime} \boldsymbol{P}_{1}+s_{2}^{\prime} \boldsymbol{P}_{2}+\ldots, \\
& \boldsymbol{Q}=q_{0}^{\prime} \boldsymbol{P}_{\text {eq }}+q_{1}^{\prime} \boldsymbol{P}_{1}+q_{2}^{\prime} \boldsymbol{P}_{2}+\ldots,
\end{aligned}
$$

where the coefficients $s_{i}^{\prime}$ and $q_{i}^{\prime}$ are defined as follows:

$$
s_{i}^{\prime}=\left(P^{-1} \boldsymbol{S}\right)_{i}, \quad q_{i}^{\prime}=\left(P^{-1} \boldsymbol{P}\right)_{i} .
$$

With the use of $s_{i}^{\prime}$ and $q_{i}^{\prime}$, Eq. (33) is represented as

$$
\begin{aligned}
& q_{0}^{\prime}=s_{0}^{\prime}=0, \\
& q_{i}^{\prime}=\frac{s_{i}^{\prime}}{-\lambda_{i}} \quad(i=1,2, \ldots) .
\end{aligned}
$$


Substituting Eqs. (43) and (44) into Eq. (41), we have

$$
\boldsymbol{Q}=\frac{s_{1}^{\prime}}{-\lambda_{1}} \boldsymbol{P}_{1}+\frac{s_{2}^{\prime}}{-\lambda_{2}} \boldsymbol{P}_{2}+\ldots
$$

Equations (42) and (45) define the procedure to obtain $\boldsymbol{Q}$ from $\boldsymbol{S}$, which is denoted as $\boldsymbol{Q}=Q(\boldsymbol{S})$.

Then, the mean first passage time approximation $\tau$ of the relaxation time is written as follows:

$$
\tau=\frac{\left\|\boldsymbol{Q}_{+}\right\|}{\left\|\boldsymbol{S}_{+}\right\|}=\frac{\left\|\boldsymbol{Q}_{-}\right\|}{\left\|\boldsymbol{S}_{-}\right\|}=\frac{\|\boldsymbol{Q}\|}{\|\boldsymbol{S}\|}=\frac{\|Q(\boldsymbol{S})\|}{\|\boldsymbol{S}\|},
$$

as discussed in the previous subsection.

Now, we consider the effect of the iterative use of this procedure. We apply the $m$-times function composition of $Q$ to $\boldsymbol{S}$, and the resultant vector $Q^{(m)}(\boldsymbol{S})$ is given by

$$
Q^{(m)}(\boldsymbol{S})=\frac{s_{1}^{\prime}}{\left(-\lambda_{1}\right)^{m}} \boldsymbol{P}_{1}+\frac{s_{2}^{\prime}}{\left(-\lambda_{2}\right)^{m}} \boldsymbol{P}_{2}+\ldots,
$$

which shows that, as $m \rightarrow \infty$, if $s_{1}^{\prime} \neq 0$, then $\boldsymbol{Q} \propto \boldsymbol{P}_{1}$, else if $s_{1}^{\prime}=\cdots=s_{i-1}^{\prime}=0$ and $s_{i}^{\prime} \neq 0$, then $\boldsymbol{Q} \propto \boldsymbol{P}_{i}$. From this, we understand that the symmetric population method can be interpreted as an inverse power method for the eigenvalue problem [53].

In Fig. 8(a), we plot the mean first passage times

$$
\tau=\frac{\left\|Q^{(m)}(\boldsymbol{S})\right\|}{\left\|Q^{(m-1)}(\boldsymbol{S})\right\|}=\frac{\left\|Q\left(Q^{(m-1)}(\boldsymbol{S})\right)\right\|}{\left\|Q^{(m-1)}(\boldsymbol{S})\right\|}
$$

for the slowest and second slowest relaxation modes with the settings of Eqs. (38) and (39), respectively. At $m=1$, both are larger than the corresponding exact relaxation times, because $\boldsymbol{S}$ of Eqs. (38) and (39) are selected so as to maximize the mean first passage times of $\|Q(\boldsymbol{S})\| /\|\boldsymbol{S}\|$. At $m=2$ and 3, the mean first passage time approximations almost converge to the corresponding relaxation times. These findings show that the mean first passage time approximations of the slowest and the second slowest relaxation times satisfy $s_{1}^{\prime} \neq 0$ and $s_{1}^{\prime}=0, s_{2}^{\prime} \neq 0$, respectively. Moreover, both of $\boldsymbol{S}$ are sufficiently accurate, so as to converge to the slowest and second slowest relaxation modes, respectively, with a few iterations. Namely, $\boldsymbol{S}$ of Eqs. (38) and (39) closely approximate the exact eigenvectors of $\boldsymbol{P}_{i}(i=1,2)$, although $\boldsymbol{S}$ are the drastic simplifications of $\boldsymbol{P}_{i}$ with very few sinks and sources.

To see the convergence of $Q^{(m)}(\boldsymbol{S})$ to the eigenvectors with the symmetric population method, we plot $Q(\boldsymbol{S})$ for the slowest and second slowest relaxation modes in Figs. 8(b) and 8(c), respectively. Comparing these graphs with Figs. 1(a) and 6. respectively, we see that $Q(\boldsymbol{S})$ with Eqs. (38) and (39) are almost the same with $\boldsymbol{P}_{1}$ and $\boldsymbol{P}_{2}$. That is, we can obtain accurate approximate eigenvectors by applying this procedure just once to the quite simplified sinks and sources of $\boldsymbol{S}$.

We remark finally that when it is difficult to set $\boldsymbol{S}$ to be in the convergence region of $\boldsymbol{P}_{2}$, we can obtain the first and second slowest relaxation modes simultaneously by iteratively applying $Q$ to two vectors that span a twodimensional subspace and orthogonalizing the vectors, as in the general diagonalization algorithms [53].
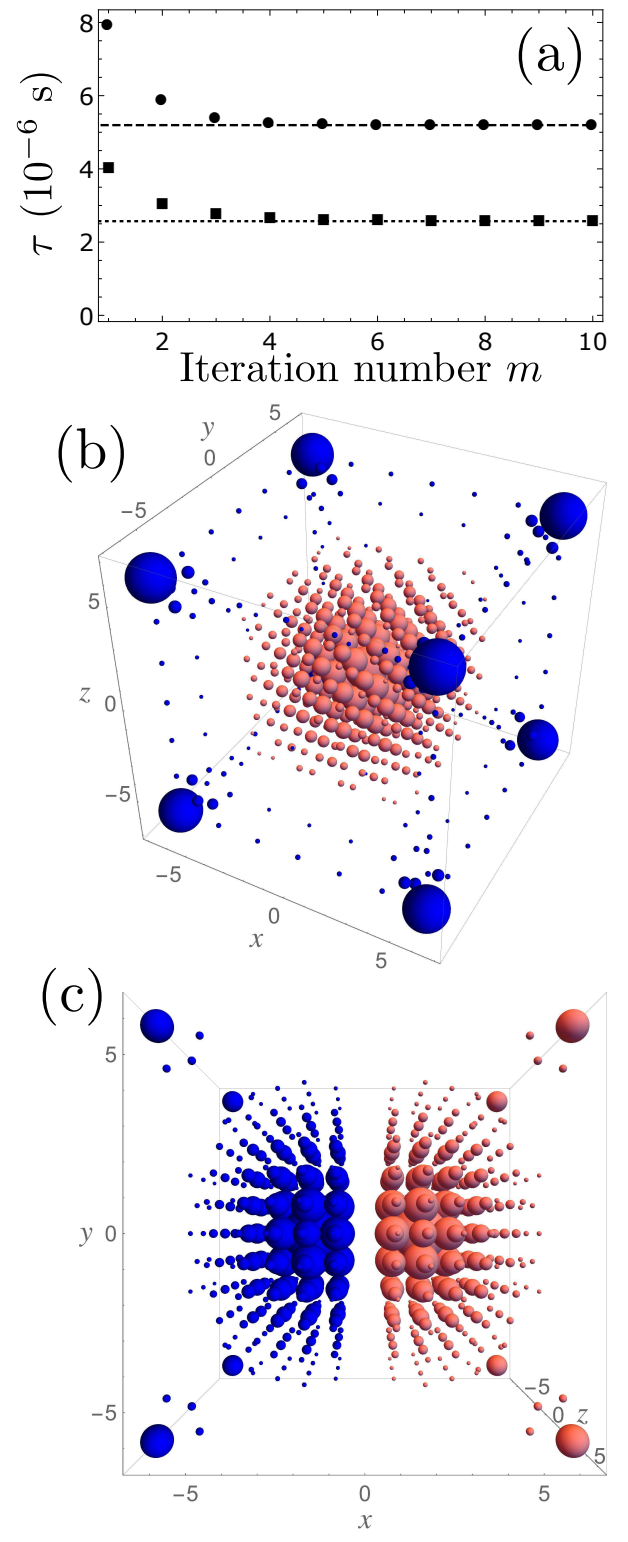

FIG. 8. The mean first passage times of the symmetric population method converge to the slowest relaxation times. (a) The mean first passage times, $\tau=\left\|Q^{(m)}(\boldsymbol{S})\right\| /\left\|Q^{(m-1)}(\boldsymbol{S})\right\|$, are plotted as a function of iteration number $m(m=$ $1,2, \ldots, 10)$ with circles for the slowest relaxation and with squares for the second slowest relaxation. The dashed and dotted lines show the values of the exact relaxation times of $1 /\left|\lambda_{1}\right|$ and $1 /\left|\lambda_{2}\right|$, respectively. $Q(\boldsymbol{S})$ are plotted in the same manner as in Fig. 1(a), for the slowest relaxation [panel (b)] and for the second slowest relaxation $[\operatorname{panel}(\mathrm{c})]$.

\section{SUMMARY}

We studied the slowest and second slowest relaxations of vacancy diffusion in a $\mathrm{KCl}$ nanocluster.

In Sec. IV we found that the slowest relaxation mode of $\boldsymbol{P}_{1}$ has cubic symmetry around the origin $(0,0,0)$, 
where the excess probability at around the origin flows into the vertices of $\left( \pm n_{L}, \pm n_{L}, \pm n_{L}\right)$ over the course of time evolution. We also found that the dominant pathways that carry large diffusive flows are classified into two types of pathways from the origin to the vertices. One is through the face centers, and the other is through the edge centers.

To understand why these pathways are selected as dominant pathways, we estimated the mean first passage times from various states to the vertex sinks. As a result, the surface diffusion turned out to be about $10^{4}$ times faster than the surface diffusion at room temperature of $k_{\mathrm{B}} T=0.03 \mathrm{eV}$. Hence, the dominant pathways turned out to be the shortest pathways to the surfaces. There, we also gave an approximation of the slowest relaxation rate $\lambda_{1}$ with the use of the mean first passage times.

Next, the reasons for the slow inner and fast surface diffusions were studied in terms of the free energy landscape. The sequences of free energies at minima and saddle points along the two types of pathways were examined. We found that the activation free energies in the inner region are about twice as large as those in the surface region, which explains the drastic slow inner diffusion. We also gave an intuitive explanation for the ratio of the activation energies that are leading terms of the activation free energies, with the use of the individual atomic energies of $V_{k}$.

In Sec. V, we considered the second slowest relaxation modes. With use of the three-dimensional plot of Fig. 6. the second relaxation modes of $\boldsymbol{P}_{2}, \boldsymbol{P}_{3}$, and $\boldsymbol{P}_{4}$ turned out to correspond to relaxation of the excesses and the deficiencies of probability in the $x$ - $y$-, and $z$-directions, respectively.
The second slowest relaxation rate of $\lambda_{2}$ is also successfully estimated by use of the mean first passage times with sinks connected to the region of $x=0$. There, the intuitive explanation for the approximate relation $\lambda_{2}, \lambda_{3}, \lambda_{4} \approx 2 \lambda_{1}$ was given in terms of the free energy landscapes along the dominant pathways.

In Sec. VI, we have developed a symmetric population method, which computes the approximate relaxation rate as the mean first passage times of particles and holes. The symmetric population method has a reasonable property in that both $\boldsymbol{P}_{i}$ and $-\boldsymbol{P}_{i}$ are the eigenvectors of the same eigenvalue. We have also shown that iterative use of the symmetric population method enables us to obtain the accurate slowest relaxation times, similarly to the inverse power method of matrix diagonalization.

In summary, we have shown that the properties of the slowest relaxation modes are reconstructed by mean first passage times in Markov state models suitably connected with sinks and sources. The mean first passage times are useful to extract the bottleneck processes buried in Markov state models. We have also shown that the formation of the bottlenecks can be understood from the physical basis of potential energy landscapes that support the networks of the Markov state models.

\section{ACKNOWLEDGMENTS}

The authors are very grateful to Shoji Tsuji and Kankikai for the use of their facilities at Kawaraya during the early stage of this study. Y.S and T.O. are supported by a Grant-in-Aid for Challenging Exploratory Research (Grant No. JP 15K13539) from the Japan Society for the Promotion of Science.
[1] M. Goldstein, J. Chem. Phys. 51, 3728 (1969).

[2] F. H. Stillinger and T. A. Weber, Science 225, 983 (1984).

[3] F. H. Stillinger, Science 267, 1935 (1995).

[4] A. Heuer, Phys. Rev. Lett. 78, 4051 (1997).

[5] L. Angelani, G. Parisi, G. Ruocco, and G. Viliani, Phys. Rev. Lett. 81, 4648 (1998).

[6] P. G. Debenedetti and F. H. Stillinger, Nature (London) 410, 259 (2001).

[7] S. Sastry, Nature (London) 409, 164 (2001).

[8] R. A. Denny, D. R. Reichman, and J.-P. Bouchaud, Phys. Rev. Lett. 90, 025503 (2003).

[9] B. Doliwa and A. Heuer, Phys. Rev. Lett. 91, 235501 (2003).

[10] G. A. Appignanesi, J. A. Rodríguez Fris, R. A. Montani, and W. Kob, Phys. Rev. Lett. 96, 057801 (2006).

[11] S. De, B. Schaefer, A. Sadeghi, M. Sicher, D. G. Kanhere, and S. Goedecker, Phys. Rev. Lett. 112, 083401 (2014).

[12] Y. Yang and B. Chakraborty, Phys. Rev. E 80, 011501 (2009).

[13] O. M. Becker and M. Karplus, J. Chem. Phys. 106, 1495
(1997).

[14] D. Shukla, C.X.Hernández, J.K. Weber, and V. S. Pande, Acc. Chem. Res.48, 414 (2015).

[15] A.B. Kolomeisky, E. B. Stukalin, and A. A. Popov, Phys. Rev. E 71, 031902 (2005).

[16] K. R. Ghusingaa, J. J. Dennehyb, and A.Singh, Proc. Natl. Acad. Sci. (U.S.A.) 114, 693 (2017).

[17] N.-V. Buchete and G. Hummer, J. Phys. Chem. B 112, 6057 (2008).

[18] G. Hummer and A. Szabo, J. Phys. Chem. B 119, 9029(2015).

[19] S. S. Cho, Y. Levy, and P. G. Wolynes, Proc. Natl. Acad. Sci. (U.S.A.) 103, 586 (2006).

[20] G. R. Bowman and V. S. Pande, Proc. Natl. Acad. Sci. (U.S.A.) 107,10890 (2010).

[21] J. Wang, R.J. Oliveira, X. Chu, P. C. Whitford, J. Chahine, W. Han, E. Wang, J. N. Onuchic, and V.B.P. Leite, Proc. Natl. Acad. Sci. (U.S.A.) 109, 15763 (2012).

[22] F. Pontiggia, D.V. Pachov, M.W. Clarkson, J. Villali, M.F. Hagan, V.S. Pande, and D. Kern, Nat. Commun. 6, 7284 (2015). 
[23] B. Zhang, W. Zheng, G.A. Papoian, and P.G. Wolynes, J. Am. Chem. Soc. 138, 8126 (2016).

[24] G. A. Breaux, R. C. Benirschke, T. Sugai, B. S. Kinnear, and M. F. Jarrold, Phys. Rev. Lett. 91, 215508 (2003).

[25] H. Haberland, T. Hippler, J. Donges, O. Kostko, M. Schmidt, and B. von Issendorff Phys. Rev. Lett. 94, 035701 (2005).

[26] K. Joshi, S. Krishnamurty, and D. G. Kanhere, Phys. Rev. Lett. 96, 135703 (2006).

[27] C. Hock, S. Straßburg, H. Haberland, B. v. Issendorff, A. Aguado, and M. Schmidt, Phys. Rev. Lett. 101, 023401 (2008).

[28] C. Hock, C. Bartels, S. Straßburg, M. Schmidt, H. Haberland, B. von Issendorff, and A. Aguado Phys. Rev. Lett. 102, 043401 (2009).

[29] T. Niiyama, S.-I. Sawada, K. S. Ikeda, and Y. Shimizu, Eur. Phys. J. D 68,1 (2014).

[30] T. Niiyama, T. Okushima, K. S. Ikeda, and Y. Shimizu, Chem. Phys. Lett. 654, 52 (2016).

[31] C. L. Brooks III, J.N. Onuchic, and D. J. Wales, Science 293, 612(2001).

[32] D. J. Wales, Energy Landscapes: Applications to Clusters, Biomolecules and Glasses (Cambridge University Press, Cambridge, UK, 2003).

[33] F. H. Stillinger, Energy Landscapes, Inherent Structures, and Condensed-Matter Phenomena (Princeton University Press, Princeton, New Jersey, 2016).

[34] An Introduction to Markov State Models and Their Application to Long Timescale Molecular Simulation, edited by G. R. Bowman, V. S. Pande, and F. Noé (Springer, New York, 2013).

[35] J.G. Kemeny and J.L. Snell, Finite Markov Chains, (Springer-Verlag, New York, 1976).

[36] J.P. Tian and D. Kannan, Stoch. Anal. Appl. 24, 685 (2006).

[37] G.R. Bowman, J. Chem. Phys. 137, 134111 (2012).

[38] D. J. Wales, Mol. Phys. 100, 3285 (2002).

[39] K. Klemm, C. Flamm, and P. F. Stadler, Eur. Phys. J. B 63, 387 (2008).

[40] T. Okushima, T. Niiyama, K. S. Ikeda, and Y. Shimizu, Phys. Rev. E 80, 036112 (2009).

[41] T. Okushima, T. Niiyama, K. S. Ikeda, and Y. Shimizu, Phys. Rev. E 76, 036109 (2007).

[42] T. Okushima, T. Niiyama, K. S. Ikeda, and Y. Shimizu,
Phys. Rev. E 97, 021301(R) (2018).

[43] T. Okushima, T. Niiyama, K. S. Ikeda, and Y. Shimizu, Phys. Rev.E 98, 032304 (2018).

[44] A. J. F. Siegert, Phys. Rev. 81, 617 (1951).

[45] J. Keilson, J. Appl. Prob. 1, 247 (1964).

[46] J. Keilson, J. Appl. Prob. 2, 405 (1965).

[47] D. A. Darling and A. J. F. Siegert, Ann. Math. Stat. 24, 624 (1953).

[48] Z. Schuss and B. J. Matkowsky, SIAM J. Appl. Math. 36, 604 (1979)

[49] B. J. Matkowsky and Z. Schuss, SIAM J. Appl. Math. 40, 242 (1981).

[50] N.G. van Kampen, Stochastic Processes in Physics and Chemistry, 3rd ed., (Elsevier, Amsterdam, 2007).

[51] D. Hartich and A. Godec, J. Stat. Mech. 2019, 024002 (2019).

[52] D. Aldous, Probability Approximations via the Poisson Clumping Heuristic (Springer-Verlag, New York, 1989).

[53] G.H. Golub and C.F. Van Loan, Matrix Computations, 4th ed. (Johns Hopkins University Press, Baltimore, MD, 2012).

[54] E.W. Montroll, K.E. Shuler, Adv. Chem. Phys. 1361 (1957).

[55] N. Deng, W. Dai, and R. M. Levy, J. Phys. Chem. B 117, 12787 (2013).

[56] W. Dai, A. M. Sengupta, and R. M. Levy, Phys. Rev. Lett. 115, 048101 (2015).

[57] Z. Q. Lee, W. Hsu, M. Lin, PLoS ONE 9, e93348 (2014).

[58] V. Tejedor, O. Bénichou, and R. Voituriez, Phys. Rev. E 80, 065104(R) (2009).

[59] Y. Kimura, Y. Saito,T. Nakada, and C. Kaito, Physica E 13, 11 (2002)

[60] M. Müller and K. Albe, Acta Mater. 55, 3237 (2007).

[61] T. Shibata, B. A. Bunker, Z. Zhang,D. Meisel, C. F. Vardeman II, and J. D. Gezelter, J. Am. Chem. Soc. 124, 11989 (2002).

[62] H. Haken, Synergetics, An Introduction: Nonequilibrium Phase Transitions and Self-Organization in Physics, Chemistry, and Biology, 3rd rev. enl. ed. (Springer, Berlin, 1983).

[63] M. P. Tosi, F. G. Fumi, J. Phys. Chem. Solids 25, 45 (1964). 